\title{
Die Konkordanzen der Carl Luythons Motetten Bellum insigne und Festa dies hodie
}

\section{The Concordances of the Motets Bellum insigne and Festa dies hodie by Carl Luython}

Jan Bilwachs / janbilwachs@seznam.cz

Ústav hudební vědy, Filozofická fakulta, Univerzita Karlova, Praha, CZ

\begin{abstract}
There are the concordances to the motet Bellum insigne from Carl Luython's collection Selectissimarum sacrarum cantionum... fasciculus primus in two partbooks from the Bohemian town Rokycany, which dates back to the turn of the $16^{\text {th }}$ and the $17^{\text {th }}$ centuries. This concordance, which was not sufficiently evaluated yet, is not just mere copy of version from Luython's earlyprint collection, but rather its reworking.

The main ambition of this paper is to consider and prove Luython's authorship of the version Bellum insigne from Rokycany. An analysis of the another motet, Festa dies hodie from the same Luython's collection and the considering of its concordance preserved in the music print Odae suavissimae commemorating the person of Jacob Chimarrhaeus should after the mutual compare with the case of Bellum insigne provide a positive answer.
\end{abstract}

\section{Key words}

Carl Luython, motet, concordance, authorship, collection, partbook, reworking, version 


\section{Selectissimarum sacrarum cantionum... fasciculus primus}

Carl Luython (1556/7 - 1620), der seit 1576 als Kammermusikus und Hoforganist, und nach dem Tod Philippe de Montes auch als Hofkomponist am Hof Rudolfs II. wirkte, vertonte während seines Dienstes eine nicht große Zahl von Motetten. Obwohl Luythons Vokalkompositionen nicht so ausgebreitet sind als die von seinen Zeitgenossen, lassen sich auch in diesem Fall einige Konkordanzen betrachten, die für Verständnis der Ausbreitung des rudolfinischen Repertoires und seines „Lebensprozesses“ in Böhmen einen großen Aussagenwert darstellen können. Um konkrete Beispiele vorzustellen, werden im vorliegenden Beitrag die Motetten Bellum insigne und Festa dies hodie vom Luythons Musikdruck Selectissimarum sacrarum cantionum... fasciculus primus mit ihren Konkordanzen in handschriftlichen und gedruckten Quellen mit der böhmischen Herkunft verglichen.

Die Musiksammlung Selectissimarum sacrarum cantionum... fasciculus primus, aus der die zwei betrachtete Motetten stammen, wurde im Jahr 1603 vom Drucker Georg Nigrin in Prag herausgegeben. ${ }^{1}$ Sie ist bis heute im kompletten Zustand als Bestandteil des fünfzehnteiligen Konvolutes in der Andreas-Möller Bibliothek in Freiberg in Sachsen überliefert. ${ }^{2}$ Sie stellt Luythons einzige Sammlung von geistlichen Motetten dar und beinhaltet auch sehr wahrscheinlich die meisten von seinen geistlichen Motetten, die er seit seinem Antritt zum Dienst an den kaiserlichen Hof im Jahr 1576 vertont hatte. ${ }^{3}$ Die Musiksammlung enthält 29 sechsstimmigen Motetten. Die vertonte Texte beziehen sich meistens auf konkrete liturgische Gelegenheiten, entweder auf Heiligenfeste, wie Bellum insigne und Festa dies hodie, oder auf temporale Feste der Oster- und Weihnachtszeit. Manche Texte aus der Sammlung enthalten auch die marianische Thematik. Neben jenen, aus liturgischen Büchern übernommenen, befinden sich in der Sammlung entweder um neue Tropen ergänzte, von mehreren Teilen verstaute, oder ganz neu verfasste Texte. ${ }^{4}$ Die Herkunft der meisten neuen Texte bleibt bisher verbergt.

Manche Merkmale der Sammlung weisen auf das Zusammenhang mit der Prager humanistischen Umgebung hin, was sowohl aus der Widmung der Sammlung dem Probst, Generalvikar, Jiří Barholdus von Breitenberg, der unter anderem auch als Literat und Dichter tätig war, ${ }^{5}$ als auch aus der vertonten Texten, deren Verfasser von der mehr komplizierten, antiken, griechischen Dichtungsformen ausging, hervorgeht. ${ }^{6}$ Als Verfas-

1 Siehe: DANĚK, Petr. Historické tisky vokálni polyfonie, rané monodie, hudebni teorie a instrumentální hudby v českých zemích do roku 1630. Praha, 2015. S. 118.

2 D-FBo, Freiberg (Sachsen), Andreas-Möller-Bibliothek, Sign. XI 847. Siehe: KADE, Otto - KADE, Reinhard. Die älteren Musikalien der Stadt Freiberg in Sachsen. Leipzig, 1888. S. 6.

3 Übersicht der Luythons Musiksammlungen ist zur Verfügung in Dissertation von Albert Smijers. Siehe: SMIJERS, Albert. Karl Luython als Motetten-Komponist. Amsterdam, 1923. S. 35-49.

4 SMIJERS, 1923. S. 52-54.

5 TRUHLÁ̌̌, Antonín - HRDINA, Karel - HEJNIC, Josef - MARTÍNEK, Jan. Rukovět humanistického básnictvi v Čechách a na Moravě: Enchiridion renatae poesis Latinae in Bohemia et Moravia cultae. 1. Praha, 1966. S. 137-139.

6 SMIJERS, 1923. S. 56. 
ser dieser neuen Texte könnte irgendwelcher von in der Umgebung des Prager Hofes wirkenden Dichtern in Betracht kommen.

\section{Luythons Bellum insigne in den Stimmbüchern von Rokycany}

In zwei handschriftlichen Stimmbüchern (AV 19a, b) von der böhmischen Stadt Rokycany, ${ }^{7}$ die außer Motetten von den in der böhmischen Umgebung wirkenden Komponisten auch Kompositionen von rudolfinischen Musikern enthalten, ist eine Fassung der Luythons Motette Bellum insigne überliefert. ${ }^{8}$ Diese Stimmbücher entstanden wahrscheinlich an der Wende des 16. und 17. Jahrhunderts. ${ }^{9}$ Sie gehören mit anderen in Rokycany überlieferten Musikalien zum Fonds, der damals unter ein Besitztum dortiger Literatenbruderschaft fiel.

Die Motette Bellum insigne ist in beiden Stimmbüchern mit Luythons Namen übergeschrieben. Dazu befindet sich in der Rubrik des Buches Bassus eine Anmerkung „De Sancto Michaele Archangelo", die sich auf den vertonten Text der Motette bezieht, der heiligem Michael Archangelus gewidmet ist. ${ }^{10}$ Michael wird in diesem, von sieben elegischen Distichen verfassten Text als Bezwinger des höllischen, aus dem stygischen Wasser stammenden Lindwurmes besingt.

Von ursprünglichen sechs Stimmen sind in den Stimmbüchern von Rokycany nur zwei überliefert. Trotzdem ist es bereits bei der ersten Untersuchung dieser Konkordanz und bei ihrem gegenseitigen Vergleich mit der gedruckten Fassung von Selectissimarum sacrarum cantionum... fasciculus primus zu bemerken, dass es nicht um eine bloße Abschrift, sondern um eine gründlich umgeschaffene Version handelt. Der vertonte Text bleibt dabei gleich und unverändert.

Auffällige musikalische Unterschiede sind bereits am Anfang, im ersten Takt der Motette zu betrachten (vgl. Beispiele 1 und 2). Die einige Notenwerte sind in beiden Stimmen gegenüber dem Druck um eine Halbe diminuiert, was wahrscheinlich auch mit der Veränderung des Metrums von Alla breve auf tempus imperfectum cum prolatione imperfecta zusammenhängt. Diese Diminution ist aber nicht wörtlich verwirklicht, denn weiter, im zweiten und dritten Takt, ist die rhythmische, und zum Teil auch melodische Bewegung von den Cantus, Quintus, und Altus in die AV 19a Stimme verändert, womit sich die aus dem Druck bekannte Struktur verliert. Einige Teile sind überdies in ihrer ursprünglichen, unveränderten Gestalt erhalten, wie sich es zum Beispiel beim zweiten und dritten Takt der Stimme AV 19b im Vergleich mit dem

7 Rokycany, Děkanská knihovna, Sign. AV 19 a, b.

8 Siehe den Katalog von Kateřina Maýrová: MAÝROVÁ, Kateřina. Hudebni prameny literátského bratrstva v Rokycanech ze XVI. a začátku XVII. století. Diplomarbeit. Univerzita Karlova. Praha, 1980. Beilage 1. S. 17.

9 MAÝROVÁ, 1980. Beilage 1. S. 3.

10 Kritische Ausgabe dieser Motette und dieses Texts ist zur Verfügung in meiner Bachelorarbeit: BILWACHS, Jan. Moteta ze sbirky Selectissimarum sacrarum cantionum... fasciculus primus Carla Luythona a jejich konkordance. Kritická edice a analýza vybraných motet. Bachelorarbeit. Univerzita Karlova. Praha, 2014. Beilage. S. III, XII-XXIX. 
Tenor von Druck feststellen lässt. Ähnliche Veränderungen befinden sich im Verlauf der ganzen Motette.

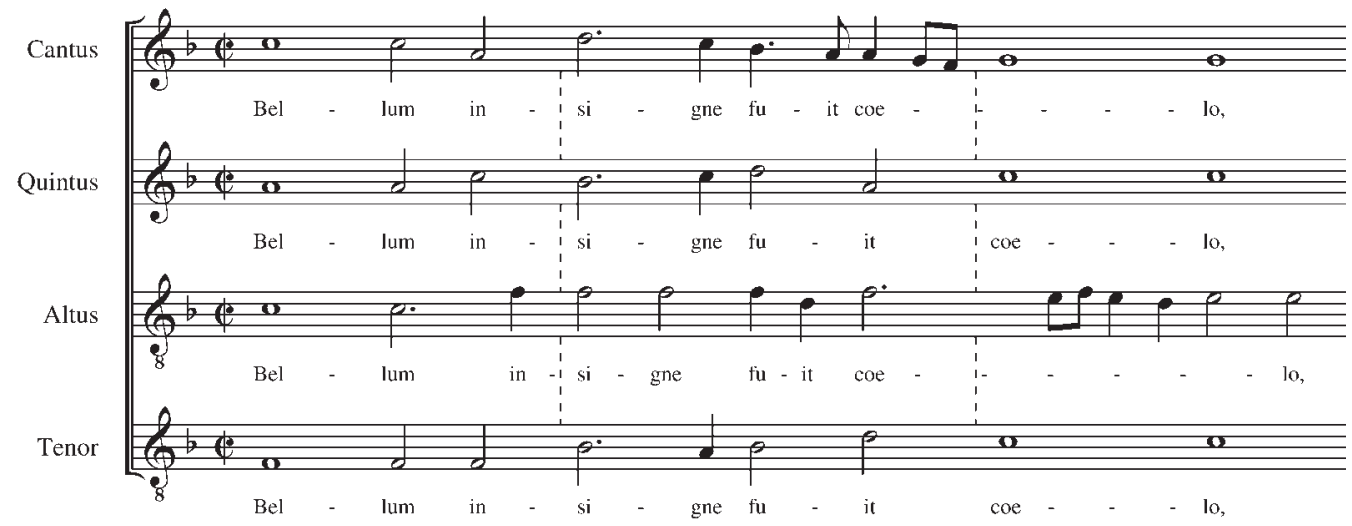

Beispiel 1. Bellum insigne in Selectissimarum sacrarum cantionum... fasciculus primus (1603), Takte $1-3 .{ }^{11}$

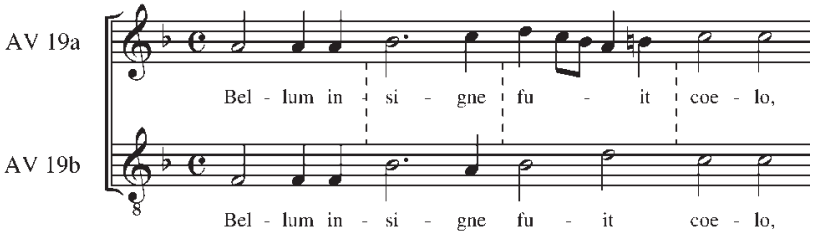

Beispiel 2. Bellum insigne, die Fassung in AV 19, Takte 1-4.

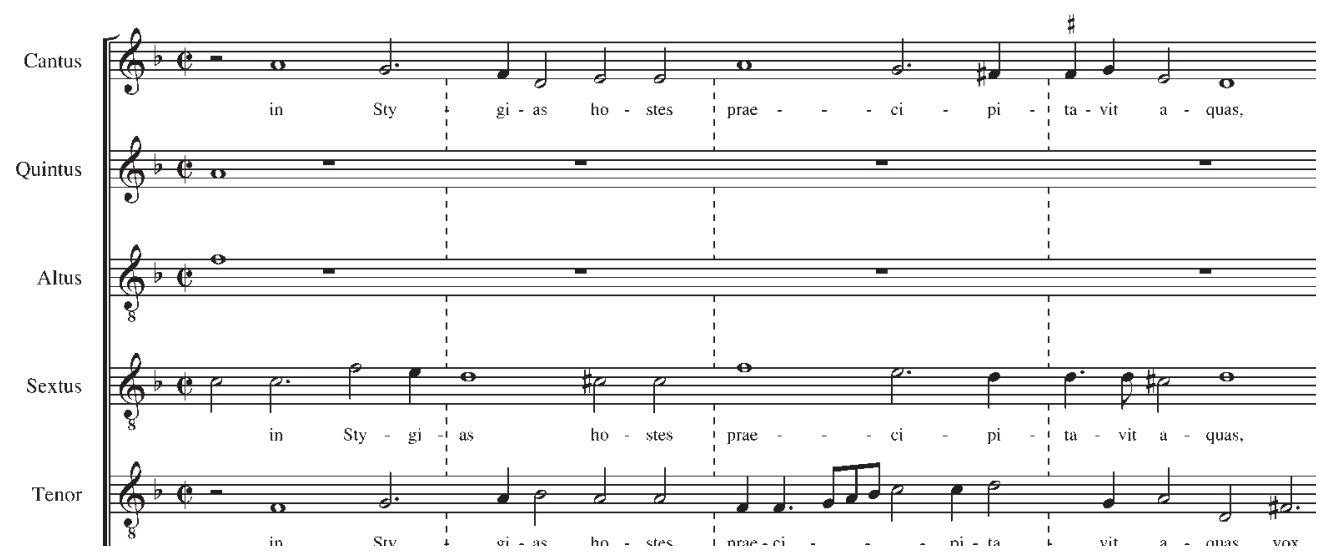

Beispiel 3. Bellum insigne in Selectissimarum sacrarum cantionum... fasciculus primus (1603), Takte 20-23.

11 Die schweigende Stimmen wurden in die Beispiele nicht aufgenommen. 


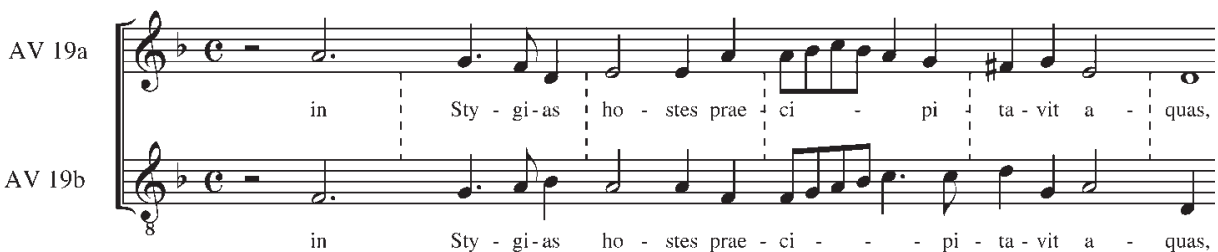

Beispiel 4. Bellum insigne, die Fassung in AV 19, Takte 27-32.

Von den Beispielen 3 und 4 ist gegenüber dem Anfang der Motette ein Wechsel in der Stimmführung zu bemerken. Die Stimme AV 19a paraphrasiert nun kein Quintus, wie in den ersten Takten, sondern Cantus. Außer der Diminution, die in den Beispielen 1 und 2 gezeigt wurde, erscheint hier auch die abweichende melodische Bewegung auf das Wort „praecipitavit“.

Die große Aufmerksamkeit verlangt das Ende der Motette, denn die letzte Takte von Bellum insigne sind komplett umgeschaffen. Während sich im Selectissimarum sacrarum cantionum... fasciculus primus die letzte Phrase nur einmal wiederholt, erscheint sie in der Fassung in AV 19 dagegen zweimal. Der melodische und rhythmische Verlauf beiden überlieferten Stimmen weist auf dieser Stelle überhaupt keine Ähnlichkeiten mit der gedruckten Fassung auf.

\section{Bellum insigne in AV 19 - Eine örtliche Bearbeitung, oder eine Autorenfassung?}

Obwohl bis zu dieser Zeit die auffälligste Unterschiede der beiden Fassungen vorgestellt wurden, ist ihre gegenseitige Zusammenhang immer unleugbar. Der vertonte Text bleibt unverändert, sowie die Gliederung der einigen Motive ist fast gleich und bis auf den Schluss der Motette sind die Motive im großen Teil miteinander ähnlich. Anhand von oben genannten Unterschieden der zwei Fassungen bietet sich eine Frage an, von wem die Fassung von Bellum insigne in den Stimmbüchern von Rokycany verfasst wurde und zwar, ob es sich um einen Gebildeten von dortiger Literatenbruderschaft handelt, der sie komplett umgeschaffen hat, oder ob unter dieser Version eine andere Fassung neben der vom Musikdruck verstanden werden soll, die auch von Luython selbst stammt.

Die erste Möglichkeit könnte aufgrund eines Vergleichs von anderen in AV 19a, b überlieferten Abschriften mit ihren wahrscheinlichen Vorlagen, oder Editionsausgaben überprüft werden. Durch die Untersuchung des Inhalts von AV 19 würde es festgestellt, ob solche Bearbeitungen dort häufiger vertreten sind und demzufolge, ob diese Bearbeitungsart eine örtliche Gewohnheit darstellt. In Betracht kommen die in den Stimmbüchern AV 19 vertretene Motetten von Jacob Handl Gallus, Orlando di Lasso, Hans Leo Hassler, oder Nicolaus Zangius. Jedoch, nach der Untersuchung dieser Überlieferungen wurden entweder keine, oder sehr kleine Unterschiede gefunden, die für überhaupt 
keine bewusste Bearbeitungen, sondern für bloße Schreibfehler halten werden können. Also, diese Hypothese über die örtliche Herkunft von Bellum insigne in den Stimmbüchern AV 19 scheint sich einstweilen unwahrscheinlich zu sein.

\section{Zwei Fassungen von Luythons Motette Festa dies hodie}

Zur Motette Festa dies hodie, die auch von Luythons Musikdruck Selectissimarum sacrarum cantionum... fasciculus primus stammt, besteht eine sehr ähnlich umgeschaffene Konkordanz, die auf die die Luythons Autorenschaft der Fassung von Bellum insigne in AV 19 betreffende Frage eine ausreichend überzeugende Antwort anbieten kann. Ihre umgeschaffene Konkordanz befindet sich in der musikalischen Festschrift Odae suavissimae..$^{12}$ Diese Festschrift wurde von Hoftrompeter Philipp Schoendorff zusammengestellt und wahrscheinlich im Jahr 1602 von Drucker Georg Nigrin in Prag herausgegeben, ${ }^{13}$ also ein Jahr vor der Herausgabe von Selectissimarum sacrarum cantionum... fasciculus primus. ${ }^{14}$ Aus ihrem Titelblatt, ${ }^{15}$ Widmungsblatt und im Tenor Stimmbuch enthaltenen Portrait, geht es zweifellos hervor, dass sie dem Jacob Chimarrhaeus gewidmet ist, der als Eleemosinarius und Direktor der Prager Hofkapelle Rudolfs II. gewirkt hatte. ${ }^{16}$ Neben Luythons Festa dies hodie befinden sich in Odae suavissimae auch Kompositionen von anderen am Hof wirkenden, oder anderenfalls mit dem Hof verbundenen Musikern. Der ganze Inhalt der Festschrift weist auf Chimarrhaeuses Persönlichkeit hin, was sowohl aus den vertonten Texten, als auch aus den häufig vorkommenden Symbolen hervorgeht. ${ }^{17}$

Aufgrund dieser Merkmale, wodurch sich Odae suavissimae charakterisieren lässt, ist es offenbar, dass es um einen ganz anderen Typ des Musikdrucks, mit ganz anderen Zielen und Zwecken handelt, als Luythons Selectissimarum sacrarum cantionum... fasciculus primus mit dem rein geistlichen Inhalt. Diese unterschiedliche Zweckbestimmung zeigt sich auch bei Festa dies hodie. Während die in Odae suavissimae vertretene Fassung eine die konkrete Persönlichkeit huldigende Motette darstellt, handelt es sich demgegenüber

12 D-Rp, Regensburg, Bischöfliche Zentralbibliothek, Proske-Musikbibliothek, Stimmen Altus, Quints, Bassus; D-W, Wolfenbüttel, Herzog August Bibliothek, Musikabteilung, Stimme Tenor.

13 Odae suavissimae bietet keine Angaben über einen Ort und ein Datum der Herausgabe. Aufgrund einer typographischen Analyse war es möglich, diese Angaben abzuschätzen. Siehe: JAKOUBKOVÁ, Petra, Typpografie hudebnich tiski̊ Jiř́ho Nigrina. Diplomarbeit. Univerzita Karlova. Praha, 2014. S. 46-48.

14 Zum Datum der Herausgabe von Odae suavissimae, siehe auch: NIEMÖLLER, Klaus Wolfgang. Die musikalische Festschrift für den Direktor der Prager Hofkapelle Kaiser Rudolfs II. 1602. In Bericht über den Internationalen Musikwissenschaftlichen Kongress. Carl Dahlhaus - Hans Joachim Marx (eds.). Bonn, 1970. S. 520.

15 Der Titel lautet: ODÆ | SVAVISSIMÆ | IN | Gratiam et Honorem | Ad modum | Reuerendi ac Illustris Dmi. | D. Iacobi Chimarrhæi Rure : | mundani S. C. M. supremi | Eleemosijnarij...[usw.] Herum dieses Titels ist im Kreis die folgende Inschrift angegeben: CHIMARRHÆE TIBI MVSICA BLANDA CANIT. OB VERÆ VIRTVTIS OPVS LAVDEMO PERENNEM.

16 NIEMÖLLER, Klaus Wolfgang. Jacob Chimarrhaeus. Ein Kölner Musiker am Habsburger Hof Rudolfs II. in Prag. In Deutsche Musik im Osten. Klaus Wolfgang Niemöller - Helmut Loos (eds.). Köln, 1994. S. 360.

17 NIEMÖLLER, 1994, S. 360. 
im zweiten Fall um eine für geistliche Zwecke bestimmte Komposition, worin heiliger Jakob besingt wird. ${ }^{18}$

Trotz der unterschiedlichen Zweckbestimmungen dieser zwei Fassungen von Festa dies hodie lässt sich andererseits ihre wechselseitige Beziehung betrachten. Was den vertonten Text anbelangt, ist seine Herkunft in der Chimarrhaeus huldigende Dichtersammlung Carmen gratulatorium aus dem Jahr 1595 zu finden. ${ }^{19}$ Aus Carmen gratulatorium wurde der Text zuerst für die wahrscheinlich früher entstandene Fassung in Odae suavissimae benutzt und erst dann wurde er umgedichtet und für die zweite Fassung verwandt. Die Jakob Chimarrhaeus gewidmete Huldigungsmotette in Odae suavissimae enthält so großen Anzahl von Parallelen mit seinem Namensgenossen, heiligem Jakob, dass der Dichter die Thematik des Texts umschaffen konnte, ohne die von vier elegischen Distichen gebildete Form zu stören. ${ }^{20}$

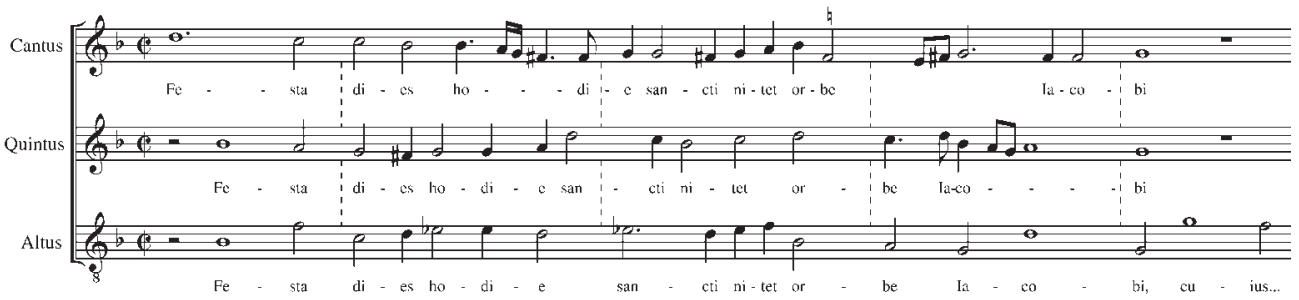

Beispiel 5. Festa dies hodie in Selectissimarum sacrarum cantionum... fasciculus primus (1603), Takte 1-5

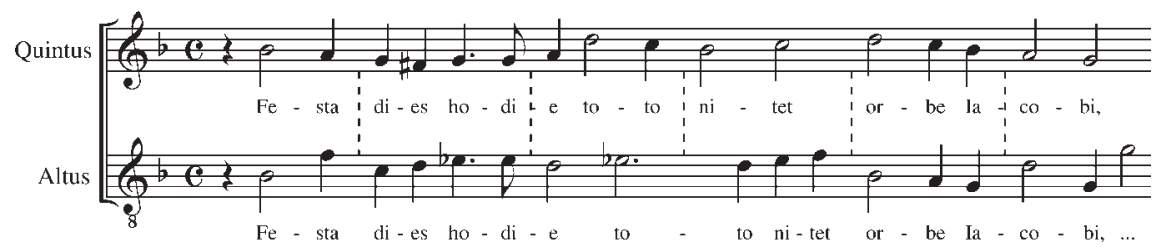

Beispiel 6. Festa dies hodie in Odae suavissimae [1602], Takte 1-6

18 Kritische Ausgabe beiden Fassungen der Motette und des Texts von Festa dies Hodie ist zur Verfügung in meiner Bachelorarbeit, siehe: BILWACHS, Jan. Moteta ze sbirky Selectissimarum sacrarum cantionum... fasciculus primus Carla Luythona a jejich konkordance. Kritická edice a analýza vybraných motet. Bachelorarbeit. Univerzita Karlova. Praha, 2014. Beilage. S. IV, XXX-XLI.

19 Michael Silies vergleicht den textlichen Inhalt von Carmen gratulatorium mit vertonten Texten in Odae suavissimae und findet, dass 17 Texte von Carmen gratulatorium stammen, siehe: SILIES, Michael. Die Motetten des Philippe de Monte (1521-1603). Göttingen, 2009. S. 238-239.

20 Erika Honisch vergleicht und analysiert in ihrer Dissertation beide Fassungen des Textes Festa dies hodie, siehe: HONISCH, Erika Supria. Sacred Music in Prague, 1580 - 1612. Dissertation. University of Chicago. Chicago - Illinois, 2011. S. 189-190. 
Für eine komplette Wiederherstellung und Berücksichtigung von Wechselunterschieden fehlen zwei Stimmen von der ursprünglichen sechsstimmigen Besetzung. Trotzdem ist es möglich festzustellen, dass die Konkordanz der Festa dies hodie von Odae suavissimae gegen die Fassung von Selectissimarum sacrarum cantionum... fasciculus primus sehr viel Unterschiede ausweist, die vorhin bei der Überlieferung der Bellum insigne in den Musikhandschriften von Rokycany betrachten werden konnten. Das Metrum der zwei Fassungen unterscheidet sich auch in diesem Fall. In Selectissimarum sacrarum cantionum... fasciculus primus wird für Festa dies hodie alla breve benutzt, während in Odae suavissimae für die gleichnamige Komposition tempus imperfectum cum prolatione imperfecta gewählt wird. In Zusammenhang damit lassen sich teilweise auch in der Fassung von Odae suavissimae die halbe Notenwerten finden und manchmal unterscheiden sich auch die Behandlung mit den Motiven und Kadenzen (vgl. Beispiele 5 und 6). Sehr auffällig ist wieder die komplette Bearbeitung der letzten Takten und die unterschiedliche Wiederholung des Textes.

\section{Zusammenfassung und Resultate}

Mit der Rücksicht auf die Bellum insigne betreffende Frage genommen, ob es sich in den Stimmbüchern von Rokycany um eine Autorenfassung handelt, verdienen sich diese zwei Vorfälle, Bellum insigne und Festa dies hodie, einen gegenseitigen Vergleich, der zum positiven Ergebnis führen sollte. Die Konkordanz von Festa dies hodie in Odae suavissimae bewies, dass sich es zwischen den zum Umfeld des Hofs näheren Quellen sehr ähnliche Bearbeitungsweise finden lassen als die in den Handschriften von Rokycany. In beiden Vorfällen war möglich fast identische Abweichungen zu betrachten, sowohl die Veränderungen der Notenwerten, als auch die Vereinfachung der Kadenzen, die Behandlung mit den Motiven, oder die komplette Umgestaltung der Enden in beiden Motetten. Unter der Voraussetzung, dass Luython beide Fassungen von Festa dies hodie vertonte, was aufgrund des Charakters und der Prager Herkunft beider Quellen mit der Motette sehr wahrscheinlich ist, lässt sich es auch voraussetzen, dass die Fassung von Bellum insigne in den Stimmbüchern von Rokycany, die von einer unbekannten Vorlage abgeschrieben worden sein kann, auch von Luython selbst vertont wurde. 


\section{Bibliography}

\section{Sources}

LUYTHON, Carl, Selectissimarum sacrarum cantionum... fasciculus primus. Pragae, 1603. RISM A/I [L 3117.

D-FBo, Freiberg (Sachsen), Andreas-Möller-Bibliothek, Sign. XI $8^{\circ} 47$.

Rokycany, Děkanská knihovna, Sign. AV 19 a, b, zwei handschriftliche Stimmbücher, bassus, discantus.

SCHOENDORFF, Philipp. Odae suavissimae. [1602]. RISM B/I/1610 ${ }^{18}$.

D-Rp, Regensburg, Bischöfliche Zentralbibliothek, Proske-Musikbibliothek, Stimmen Altus, Quints, Bassus.

D-W, Wolfenbüttel, Herzog August Bibliothek, Musikabteilung, Stimme Tenor.

\section{Literature}

BILWACHS, Jan. Moteta ze sbirky Selectissimarum sacrarum cantionum... fasciculus primus Carla Luythona a jejich konkordance. Kritická edice a analýza vybraných motet. Bachelorarbeit. Univerzita Karlova. Praha, 2014.

DANĚK, Petr. Historické tisky vokálni polyfonie, rané monodie, hudebni teorie a instrumentálni hudby $v$ českých zemich do roku 1630. Praha, 2015.

HONISCH, Erika Supria. Sacred Music in Prague, 1580-1612. Dissertation. University of Chicago. Chicago - Illinois, 2011.

JAKOUBKOVÁ, Petra, Typografie hudebních tisků Jiř́ho Nigrina. Diplomarbeit. Univerzita Karlova. Praha, 2014.

KADE, Otto - KADE, Reinhard. Die älteren Musikalien der Stadt Freiberg in Sachsen. Leipzig, 1888.

MAÝROVÁ, Kateřina. Hudebni prameny literátského bratrstva v Rokycanech ze XVI. a začátku XVII. stoleti. Diplomarbeit. Univerzita Karlova. Praha, 1980.

NIEMÖLLER, Klaus Wolfgang. Die musikalische Festschrift für den Direktor der Prager Hofkapelle Kaiser Rudolfs II. 1602. In Bericht über den Internationalen Musikwissenschaftlichen Kongress. Carl Dahlhaus - Hans Joachim Marx (eds.). Bonn, 1970.

NIEMÖLLER, Klaus Wolfgang. Jacob Chimarrhaeus. Ein Kölner Musiker am Habsburger Hof Rudolfs II. in Prag. In Deutsche Musik im Osten. Klaus Wolfgang Niemöller - Helmut Loos (eds.). Köln, 1994.

SILIES, Michael. Die Motetten des Philippe de Monte (1521-1603). Göttingen, 2009.

SMIJERS, Albert. Karl Luython als Motetten-Komponist. Amsterdam, 1923.

TRUHLÁ̌́, Antonín - HRDINA, Karel - HEJNIC, Josef - MARTÍNEK, Jan. Rukovět humanistického básnictvi v Čechách a na Moravě: Enchiridion renatae poesis Latinae in Bohemia et Moravia cultae. Praha, 1966-2011. 
